\title{
Gastric Signet Ring Cell Carcinoma Arising within 10 Months Interval After Chemotherapy of Primary Gastric Diffuse Large B-Cell Lymphoma: A Case Report
}

ISSN: 2637-7632

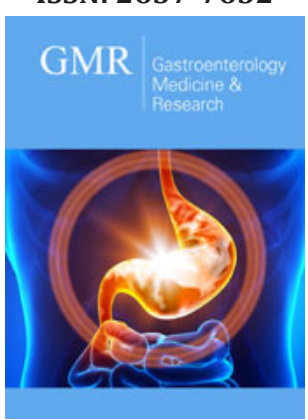

*Corresponding author: Hui Liang, Department of GI Surgery, Zhuhai People's Hospital, Zhuhai, China

Submission: 佂 December 09, 2019

Published: 㭗January 13, 2020

Volume 4 - Issue 2

How to cite this article: Xinbo A, Hui Liang. Gastric Signet Ring Cell Carcinoma Arising within 10 Months Interval After Chemotherapy of Primary Gastric Diffuse Large B-Cell Lymphoma: A Case Report. Gastro Med Res. 4(2). GMR.000583. 2020. DOI: $10.31031 /$ GMR.2020.04.000583

Copyright@ Hui Liang, This article is distributed under the terms of the Creative Commons Attribution 4.0 International License, which permits unrestricted use and redistribution provided that the original author and source are credited.

\author{
Xinbo $\mathrm{Ai}^{1}$ and Hui Liang ${ }^{2 *}$ \\ ${ }^{1}$ Department of Gastroenterology, Zhuhai People's Hospital, Zhuhai, China \\ ${ }^{2}$ Department of GI Surgery, Zhuhai People's Hospital, Zhuhai, China
}

\begin{abstract}
Background: Gastric signet ring cell carcinoma could occur in few cases of primary gastric lymphoma. It is less common seen after chemotherapy of gastric diffuse large B-cell lymphoma. Herein we present a case with gastric signet ring cell carcinoma occurring within the shortest interval of chemotherapy of gastric diffuse large B-cell lymphoma.
\end{abstract}

Case presentation: A 60-year-old male patient presented with intermittent upper abdominal pain, he underwent gastroscopy and there were multiple ulcerations in the stomach and biopsy showed diffused large B-cell lymphoma (DLBCL). The patient received chemotherapy, gastroscopy follow up was done after two months, three months, six months respectively. However, gastroscopic biopsy showed gastric signet ring cell carcinoma (SRC) within 10 months interval since he received the chemotherapy.

Conclusion: Our case highlights the importance of gastroscopy follow-up after chemotherapy of gastric DLBCL in the long term. Combination with chromoendoscopy, magnified endoscopy and target biopsy is crucial. Endoscopists need to be aware of potential risk of gastric adenocarcinoma occurring with primary gastric lymphoma.

Keywords: Gastroscopy; Gastric lymphoma; Adenocarcinoma; Surveillance

Abbreviations: DLBCL: Diffuse Large B Cell Lymphoma; GA: Gastric Adenocarcinoma; PGL: Primary Gastrointestinal Lymphoma; SRC: Signet Ring Cell Carcinoma; WLI: White Light Imaging; PET-CT: Positron Emission Tomography Computed Tomography; HE: Hematoxylin and Eosin Staining

\section{Introduction}

The simultaneous association of gastric carcinoma (GA) with primary gastric lymphoma (PGL) is quite rare. Ten patients developed metachronous GA after treatment of PGL. They stated that the risk of metachronous GA was higher in the patients with age over 60 years, DLBCL, Helicobacter pylori infection, chemotherapy treatment [1]. Therefore, we report a case of gastric SRC occurring after gastric DLBCL chemotherapy, and the shortest interval time was 10 months from gastric DLBCL to SRC.

\section{Case Presentation}

A 60-year-old male patient presented with intermittent upper abdominal pain for 6 months. The patient had no melena or weight loss. He received gastroscopy (EPK-i7000, Pentax, Japan) and there were multiple gastric ulcerations in the stomach (Figure 1). Furthermore, biopsy showed intramucosal lymphoid epithelial lesions (LEL) with HE is staining. Meanwhile, urea breath test showed strongly positive. Consequently, Immunohistopathology (IHC) showed diffused positive with CD20, CD79a (Figure 2A, 2B \& 2C). Our experienced pathologist made the diagnosis of gastric DLBCL. Then the patient was admitted to Department of Hematology, PET-CT indicated that local gastric wall thickening. It was IEA stage based upon Lugano stage, the patient received Helicobacter Pylori eradication and CHOP chemotherapy (CTX 1.1+THP 75mg+VDS 4mg+PHD 90 mgd1-5). The patient underwent gastroscopy surveillance. Gastroscopy showed gastric scar and numerous inflammatory cells infiltration proved by biopsy after two months, three months, six months respectively (Figure 3A, 3B \& 3C). Endoscopist 
performed gastroscopy ten months later after chemotherapy and we could find out a superficially depressed and reddish lesion (0IIc) at the lesser curve of gastric body with white light imaging (WLI). The depressed lesion was approximately $0.8 * 1.0 \mathrm{~cm}$ in size, and demarcate line was clear with chromoendoscopy (Figure 4A \& 4B). Magnified endoscopy showed abnormal micro vessels and irregular micro surface pattern (Figure 4C). Biopsy showed some signet ring cells infiltration with atrophic gastritis (Figure 4D). The patient was transferred to Department of General Surgery and laparoscopic resection specimen showed gastric intramucosal signet ring cell carcinoma. Surgical specimen showed no vessel or lymphoma metastasis. Definitely the patient's prognosis was very good, but he still needs to under surveillance in the long run.
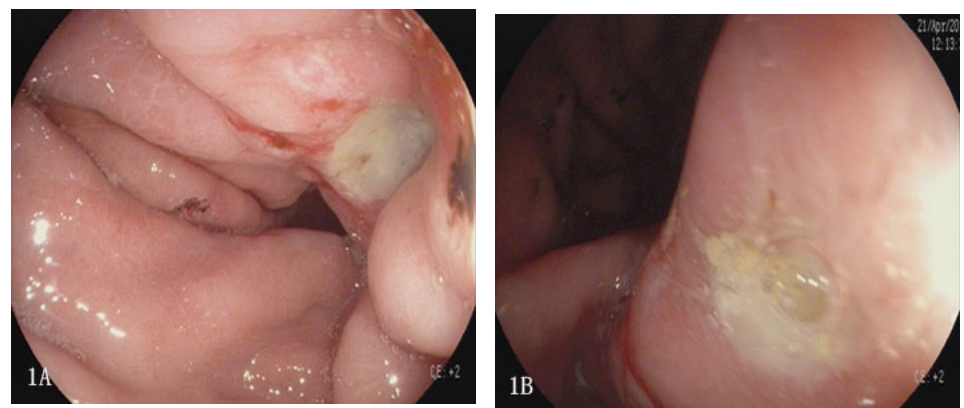

Figure 1: Multiple gastric ulcerations in the stomach (1A,1B).
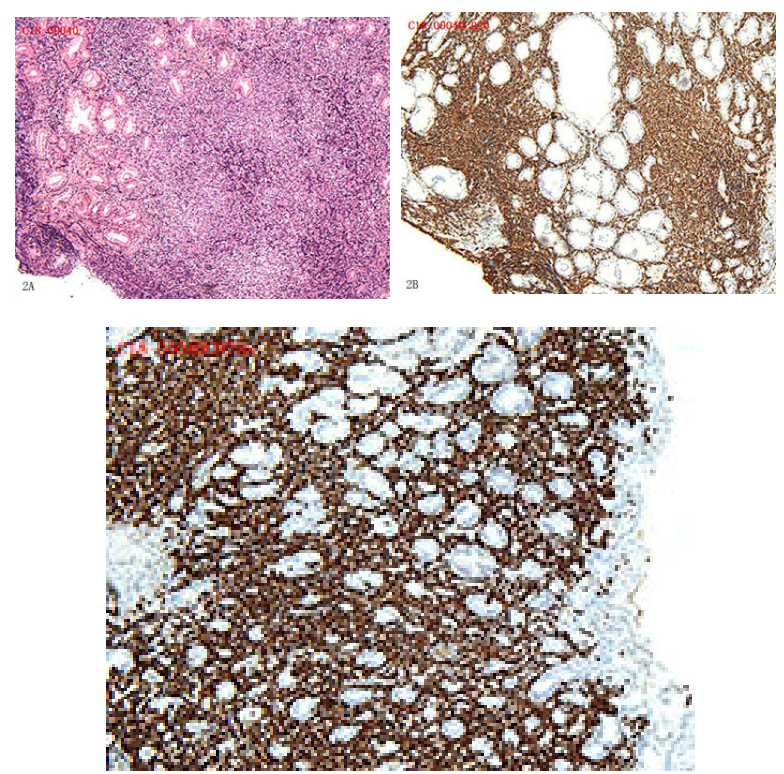

Figure 2: 2A Biopsy showing intramucosal lymphoid epithelial lesions (LEL) with HE staining. 2B, 2C showing diffused positive with CD20, CD79a.
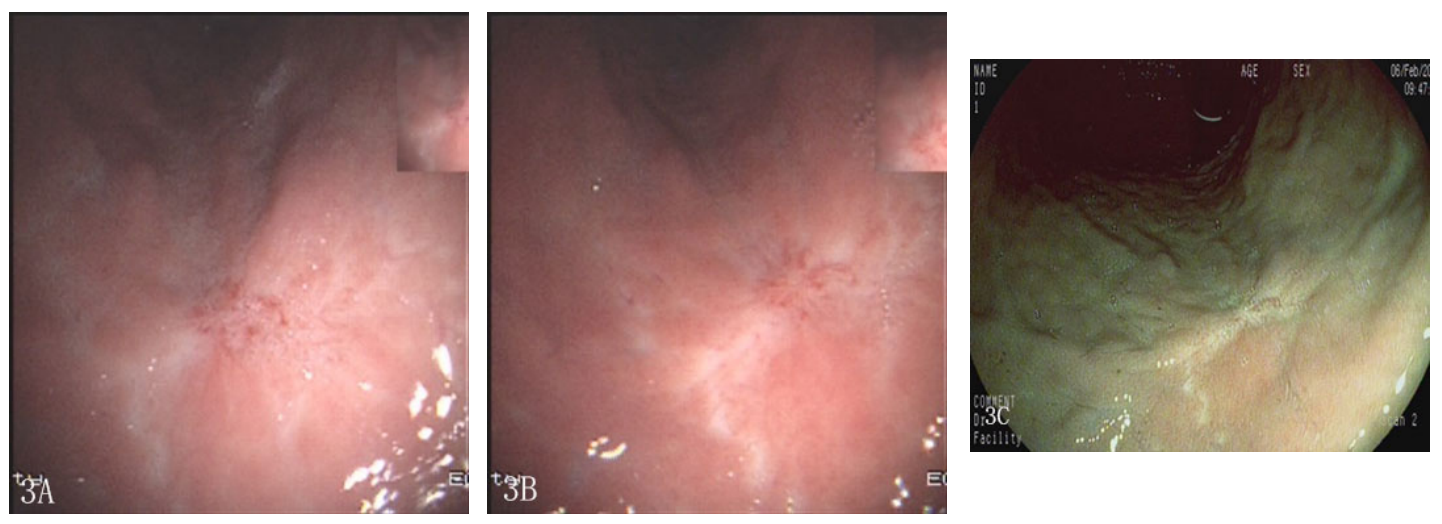

Figure 3: Gastroscopy showing gastric scar and numerous inflammatory cells infiltration proved with biopsy after 2 months, 3 months, 6 months respectively. 

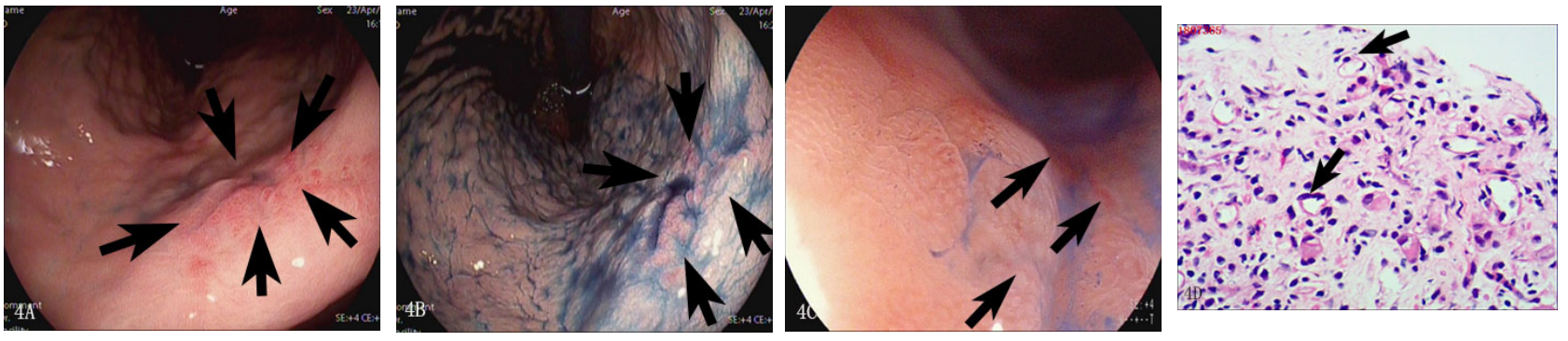

Figure 4:

(4A): WLI showing lesser curve of gastric body superficially depressed lesion approximately $0.8 * 1.0$ in size (Arrow).

(4B): Chromoendoscopy showing 0-IIc lesion with clear demarcation line. (Arrow).

(4C): Magnified endoscopy showing slightly abnormal micro vessels and regular micro surface. (Arrow).

(4D): Biopsy showing intramucosal signet ring cells carcinoma infiltrations. (HE 20×10) (Arrow).

\section{Discussion and Conclusion}

There were few case reports of synchronous GA and PGL not only in Japan but also in Europe [1-3]. Regarding synchronous GA and PGL, Ishihama et al. [4] reported that GA was seen synchronously in $3.3 \%$ of PGL patients. Inaba et al. [1] have seen that there were $10(7.2 \%)$ metachronous GA patients after treatment of PGLs, and the shortest interval from gastric DLBCL to gastric SRC was 42.6 months. However, outpatient's interval from gastric DLBCL to gastric SRC was just 10 months, it seems that the interval time was the shortest as far as we know. With regard to its potential pathogenesis from gastric DLBCL to GA, some postulated that gastric mucosal damages due to chemotherapy, while others believed that GA remained undetected at the time of PGL because GA was very small or minute cancer $[4,5]$. Some have argued about whether Helicobacter pylori (HP) needs to be eradicated for gastric DLBCL. The Japanese study has demonstrated that some patients with localized, HP-positive gastric pure (de novo) DLBCL achieved durable pCR after HP eradication therapy [6,7]. It means HP eradication is quite effective for some patients with gastric DLBCL. Our patient received HP eradication therapy absolutely. Whether HP eradication is global consensus of gastric DLBCL management still needs further clinical research. The case reminds us we need to be aware of high risk of GA incidence after gastric DLBCL chemotherapy. We need to pay attention to rigorous gastroscopy surveillance. Combination of WLI, chromoendoscopy or magnified endoscopy is of great value.

\section{Declarations}

\section{Acknowledgement}

We are very thankful to Prof. Zhu Jin (Department of Pathology, Peking University Hospital) for her pathological assistance.

\section{Availability of data and materials}

All data used and analysed of the case can be required from the corresponding author.

\section{Competing interests}

The authors declare that they have no competing interests.

\section{Funding}

No funding was obtained for this study.

\section{References}

1. Inaba K, Kushima R, Murakami N, Kuroda Y, Harada K, et al. (2013) Increased risks of gastric adenocarcinoma after treatment of primary gastric large B-cell lymphoma. BMC Cancer 13: 1-6.

2. Nakamura S, Aoyagi K, Iwanaga S, Yao T, Tsuneyoshi M, et al. (1997) Synchronous and metachronous primary gastric lymphoma and adenocarcinoma: A clinicopathological study of 12 patients. Cancer 79(6): 1077-1085.

3. Capelle LG, de Vries AC, Looman CW, Casparie MK, Boot H, et al. (2008) Gastric MALT lymphoma: Epidemiology and high adenocarcinoma risk in a nation-wide study. Eur J Cancer 44(16): 2470-2476.

4. Ishihama T, Kondo H, Saito D, Yamaguchi H, Shirao K, et al. (1997) Clinicopathological studies on coexisting gastric malignant lymphoma and gastric adenocarcinoma: Report of four cases and review of the Japanese literature. Jpn J Clin Oncol 27(2): 101-106.

5. Hamaloglu E, Topaloglu S, Ozdemir A, Ozenc A (2006) Synchronous and metachronous occurrence of gastric adenocarcinoma and gastric lymphoma: A review of the literature. World J Gastroenterol 12(22): 3564-3574.

6. Haruma K, Suzuki T, Tsuda T, Yoshihara M, Sumii K, et al. (1991) Evaluation of tumor growth rate in patients with early gastric carcinoma of the elevated type. Gastrointest Radiol 16(4): 289-292.

7. Sugimoto M, Kajimura M, Sato Y, Hanai H, Kaneko E, et al. (2001) Regression of primary gastric diffuse large B-cell lymphoma after eradication of Helicobacter pylori. Gastrointest Endosc 54(5): 643-645.

For possible submissions Click below: 